\title{
Erratum to: Minimizing total tardiness on parallel machines with preemptions
}

\author{
Svetlana A. Kravchenko • Frank Werner
}

Published online: 26 January 2013

(C) Springer Science+Business Media New York 2013

\begin{abstract}
In this note, we correct a mistake which was made in the paper "Minimizing Total Tardiness on Parallel Machines with Preemptions" (see J Schedul 15:193-200, 2012).
\end{abstract}

Keywords Parallel machines - Total tardiness ·

Preemptions · NP-hardness

\section{Introduction}

In Sect. 3 of paper (Kravchenko and Werner 2012), a mistake in the NP-hardness proof for the problem $P|\operatorname{pmtn}| \sum T_{j}$ was made. A counterexample was given in Prot et al. (2012). In Sect. 2 of this note, we present a corrected proof. In Sect. 3 , we give some further remarks.

\section{Corrected proof}

In this section, we show that problem $P \mid$ pmtn $\mid \sum T_{j}$ is NP-hard, i.e., we consider the problem of scheduling $n$ jobs on $m$ machines $\{1, \ldots, m\}$ with preemptions to minimize the total tardiness value $\sum_{j=1}^{n} T_{j}$. Thus, for each job $j$, we know in advance its processing time $p_{j}$ and its due date $d_{j}$.

The online version of the original article can be found under doi:10.1007/s10951-010-0198-5.

S. A. Kravchenko

United Institute of Informatics Problems, Surganova St. 6, 220012

Minsk, Belarus

F. Werner $(\varangle)$

Fakultät für Mathematik, Otto-von-Guericke-Universität,

39106 Magdeburg, Germany

e-mail: frank.werner@mathematik.uni-magdeburg.de
The proof is obtained by a reduction from PARTITION: Given a set of positive integers $a_{1}, \ldots, a_{k}, b$ with $\sum_{i=1}^{k} a_{i}=$ $2 b$. Does there exist a subset $I \subseteq\{1, \ldots, k\}$ such that $\sum_{i \in I} a_{i}=b$ ?

PARTITION is ordinary NP-hard (Karp 1972), and it can be shown that it remains so under the assumption that $3 k<$ $b / 2$. To see this, it is sufficient to replace $a_{1}, \ldots, a_{k}, b$ by $6 k a_{1}, \ldots, 6 k a_{k}, 6 k b$.

Given any instance of PARTITION, we define a corresponding instance of the problem $P \mid$ pmtn $\mid \sum T_{j}$ as follows. Let $n=3 k-1$ and $m=k$. We set $L=10 b^{3}$. There are three classes of jobs:

- the class of $a$-jobs comprises $k$ jobs with processing times $a_{i}$ and due dates $d_{i}=L$ for $i=1, \ldots, k$;

- the class of $b a$-jobs comprises $k$ jobs with processing times $9 b^{3}+a_{i} b^{2}-a_{i}$ and due dates $d_{i}=L-a_{i}$ for $i=1, \ldots, k$;

- the class of $b$-jobs comprises $k-1$ jobs with processing times $b^{3}$ and due dates $b^{3}$.

We claim that PARTITION has a solution if and only if there exists a schedule with $\sum T_{j} \leq b^{3}+b$.

First, we show that, if PARTITION has a solution, then for the corresponding instance of the problem $P|\operatorname{pmtn}| \sum T_{j}$ a schedule exists with $\sum T_{j} \leq b^{3}+b$. Without loss of generality, suppose that $\left\{a_{1}, \ldots, a_{z}\right\}$ is a solution for PARTITION, i.e., we suppose that $\sum_{i=1}^{z} a_{i}=b$ holds. Then:

1. we schedule each $b a$-job $i, i=1, \ldots, k$, with processing time $9 b^{3}+a_{i} b^{2}-a_{i}$ for $9 b^{3}-a_{i}$ time units in the interval $\left[b^{3}, L-a_{i}\right]$ on machine $i$, and each $b a$ job $i, i=1, \ldots, z$, for $a_{i} b^{2}$ time units in the interval $\left[L, L+a_{i} b^{2}\right]$ on machine $i$ 
Fig. 1 Scheduling the jobs for the given partition

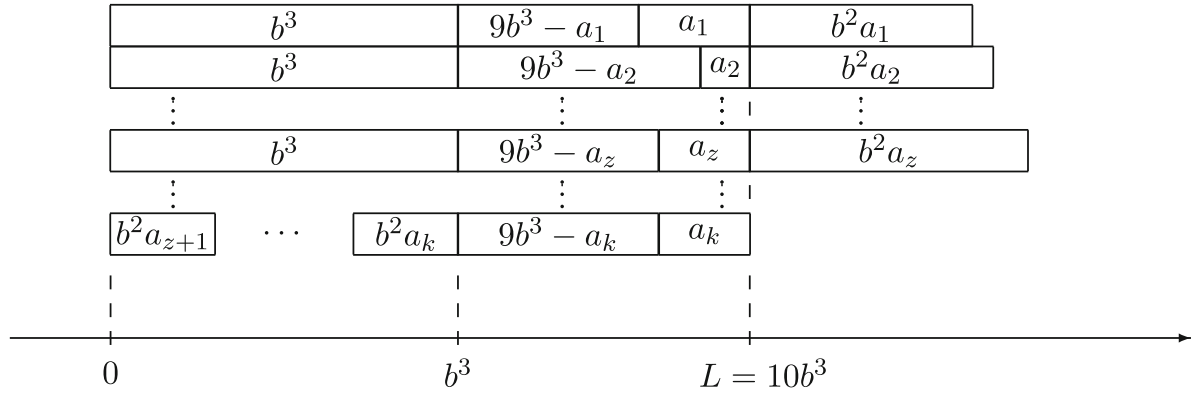

2. we schedule each $a$-job $i, i=1, \ldots, k$, with processing time $a_{i}$ for $a_{i}$ time units in the interval $\left[L-a_{i}, L\right]$ on machine $i$;

3. we schedule each $b$-job $i, i=1, \ldots, k-1$, with processing time $b^{3}$ for $b^{3}$ time units in the interval $\left[0, b^{3}\right]$ on machine $i$; and

4. we schedule the remaining parts of the $b a$-jobs $i, i=$ $z+1, \ldots, k$, in the interval $\left[0, b^{3}\right]$ on machine $k$, see Fig. 1.

Note that after steps $1-3$, all $a$-jobs, all $b$-jobs, and all $b a$-jobs for $i=1, \ldots, z$ are completely scheduled whereas for $i=$ $z+1, \ldots, n$, each $b a$-job is scheduled for $9 b^{3}-a_{i}$ time units. Thus, before step 4 , the only unscheduled jobs are the $b a$-jobs for $a_{i} b^{2}$ time units, $i=z+1, \ldots, n$.

Since, before step 4, machine $k$ is idle for the time interval $\left[0, b^{3}\right]$ and $\sum_{i=z+1}^{n} a_{i} b^{2}=b^{3}$ holds, step 4 completes the construction of a feasible schedule.

Besides, for the constructed schedule we obtain

$$
\begin{gathered}
\sum_{b \text {-jobs }} T_{j}=0, \quad \sum_{a \text {-jobs }} T_{j}=0, \quad \sum_{\substack{b a \text {-jobs } \\
j \in\{1, \ldots, z\} \\
j \in\{\text {-jobs } \\
j \in\{z+1, \ldots, n\}}} T_{j}=b^{3}+b, \\
\sum_{j}=0 .
\end{gathered}
$$

Thus, for the constructed schedule $\sum T_{j}=b^{3}+b$ holds.

Next, we show that, if there exists a schedule for which $\sum T_{j} \leq b^{3}+b$ holds, then PARTITION has a solution. Consider a schedule, say $s$, with $\sum T_{j} \leq b^{3}+b$.

The $b$-jobs have to be scheduled within the time interval $\left[0,2 b^{3}+b\right]$, since otherwise inequality $\sum T_{j}>b^{3}+b$ holds. Thus, after $L$ only $a$-jobs and $b a$-jobs can be scheduled.

All $a$-jobs have to be scheduled before their due dates, i.e., before the time $L=10 b^{3}$. To see this, consider a $b a$-job, say $f$, with the maximal completion time $C_{f}$ among all $b a$-jobs. There are two cases:

Case 1. If there is an $a$-job scheduled (partially or completely) within the interval $\left[C_{f},+\infty\right.$ [, one can switch this part of the $a$-job with the $b a$-job $f$ scheduled within the interval $\left[2 b^{3}+b, 9 b^{3}\right]$. The value $\sum T_{j}$ does not increase.

Case 2. However, if there are no $a$-jobs scheduled within the interval $\left[C_{f},+\infty[\right.$, take any $a$-job processed within the interval $\left[L, C_{f}\right.$ [ for some time, say for $\Delta$ time units. Denote this part of the $a$-job by $a_{\Delta}$. Now one can take the part of the $b a$-job $f$ processed within the interval $\left[2 b^{3}+b, 9 b^{3}\right]$ for $\Delta$ time units. Denote this part of the job by $f_{\Delta}$. Finally, one can replace $f_{\Delta}$ by $a_{\Delta}$, and schedule $f_{\Delta}$ in the interval $\left[C_{f}, C_{f}+\Delta\right]$. The value of $\sum T_{j}$ does not increase.

As a result, we obtain a new schedule, say $s^{\prime}$, where all $a$-jobs are processed before $L$ and the completion time of the $b a$-job $f$ is $C_{f}+X$, where $X$ is the total amount of the processing of $a$-jobs, scheduled after $L$ in $s$. Since the completion times are known for all jobs, the schedule $s^{\prime}$ can be constructed without applying the described transformation, but using a network flow model (Horn 1974) with a vertex for each job and for each completion time.

Thus, after the time $L=10 b^{3}$, only $b a$-jobs can be scheduled. Denote this set of jobs by $A$, i.e., $A=\{i \mid$ $i$ is a $b a$-job and $\left.C_{i} \geq L\right\}$. Denote by $l_{i}$ the total length of a $b a$-job $i$, scheduled after $L$.

Moreover, since the sum of all processing times is $10 b^{3} k+$ $b^{3}$, we have $\sum_{i \in A} l_{i} \geq b^{3}$ and therefore, all $b$-jobs have to be scheduled not in the interval $\left[0,2 b^{3}+b\right]$ but in the interval $\left[0, b^{3}+b\right]$.

Thus, within the interval $\left[b^{3}+b, 10 b^{3}\right]$, only $b a$-jobs and $a$-jobs can be scheduled. Since the total length of the $a$-jobs is $2 b$, it follows that each $b a$-job has to be processed in the interval $\left[b^{3}+b, 10 b^{3}\right]$ for at least $9 b^{3}-3 b$ time units and thus, inequality $l_{i} \leq p_{i}-9 b^{3}+3 b$ holds, and since $p_{i}=$ $9 b^{3}+a_{i} b^{2}-a_{i}$, we obtain the inequality $l_{i} \leq a_{i} b^{2}+3 b-a_{i}$, i.e., $l_{i} \leq a_{i} b^{2}+3 b$ holds.

Now, by assumption, we have $b^{3}+b \geq \sum_{i \in A} T_{i}$. Since $\sum_{i \in A} T_{i} \geq \sum_{i \in A} a_{i}+\sum_{i \in A} l_{i}$ and $\sum_{i \in A} l_{i} \geq b^{3}$, we obtain

$b^{3}+b \geq \sum_{i \in A} T_{i} \geq \sum_{i \in A} a_{i}+\sum_{i \in A} l_{i} \geq \sum_{i \in A} a_{i}+b^{3}$

and therefore, $\sum_{i \in A} a_{i} \leq b$ holds.

Moreover, since inequalities $\sum_{i \in A} l_{i} \geq b^{3}$ and $l_{i} \leq$ $a_{i} b^{2}+3 b$ hold, we get

$b^{3} \leq \sum_{i \in A} l_{i} \leq \sum_{i \in A}\left(a_{i} b^{2}+3 b\right)$. 
Now, we have $\sum_{i \in A}\left(a_{i} b^{2}+3 b\right)=\left(\sum_{i \in A} a_{i}\right) b^{2}+|A| 3 b$. Since $|A| \leq k$, then

$$
b^{3} \leq\left(\sum_{i \in A} a_{i}\right) b^{2}+3 k b \text {. }
$$

Thus, one can see that, if $3 k<b / 2$, then inequality $\sum_{i \in A} a_{i} \geq b$ holds.

Hence, we obtain $\sum_{i=1}^{z} a_{i}=b$, i.e., the set of $b a$-jobs completed after time point $L$ defines the solution for PARTITION.

\section{Remarks}

In Kravchenko and Werner (2012), the polynomial transformation from PARTITION to the problem $P \mid r_{j}, p_{j}=$ $p$, pmtn $\mid \sum T_{j}$ substantially used the decision version of the problem $P \mid$ pmtn $\mid \sum T_{j}$. In fact, the decision version of the problem $P \mid$ pmtn $\mid \sum T_{j}$ was polynomially reduced to the decision version of the problem $P \mid r_{j}, p_{j}=$ $p$, pmtn $\mid \sum T_{j}$. In the corrected proof, we changed the decision version of the problem $P \mid$ pmtn $\mid \sum T_{j}$. However, it is easy to see that the new decision version of the problem $P \mid$ pmtn $\mid \sum T_{j}$ can be polynomially transformed to the problem $P \mid r_{j}, p_{j}=p$, pmtn $\mid \sum T_{j}$ using the same scheme given in Kravchenko and Werner (2012).

Thus, both problems $P|\operatorname{pmtn}| \sum T_{j}$ and $P \mid r_{j}, p_{j}=$ $p$, pmtn $\mid \sum T_{j}$ are NP-hard.

\section{References}

Horn, W. A. (1974). Some simple scheduling algorithms. Naval Research Logistics Quarterly, 21, 177-185.

Karp, R. M. (1972). Reducibility among combinatorial problems. In R. E. Miller \& J. W. Thatcher (Eds.), Complexity of computer computations (pp. 85-103). New York: Plenum Press.

Kravchenko, S. A., \& Werner, F. (2012). Minimizing total tardiness on parallel machines with preemptions. Journal of Scheduling, 15, 193-200.

Prot, D., Bellenguez-Morineau, O., \& Lahlou, C. (2012). A note on the paper "Minimizing total tardiness on parallel machines with preemptions" by Kravchenko and Werner (2012). Journal of Scheduling doi:10.1007/s10951-012-0283-z. 